\title{
Introduction to radioactive materials
}

\author{
Askari Mohammad Bagher ${ }^{1,}$, Mirzaei Vahid ${ }^{2}$, Mirhabibi Mohsen ${ }^{3}$ \\ ${ }^{1}$ Department of Physics, Payame Noor University, PO Box 19395-3697 Tehran, Iran \\ ${ }^{2}$ Faculty of Physics, Shahid Bahonar University, P.O. Box 76175, Kerman, Iran \\ ${ }^{3}$ Department of Physics, Payame Noor University, PO Box 19395-3697 Tehran, Iran
}

\section{Email address:}

MB_Askari@yahoo.com (M. B. Askari)

\section{To cite this article:}

Askari Mohammad Bagher, Mirzaei Vahid, Mirhabibi Mohsen. Introduction to Radioactive Materials. International Journal of Renewable and Sustainable Energy. Vol. 3, No. 3, 2014, pp. 59-67. doi: 10.11648/j.ijrse.20140303.13

\begin{abstract}
Radioactivity is a part of nature. Everything is made of atoms. Radioactive atoms are unstable; that is, they have too much energy. When radioactive atoms spontaneously release their extra energy, they are said to decay. All radioactive atoms decay eventually, though they do not all decay at the same rate. After releasing all their excess energy, the atoms become stable and are no longer radioactive. The time required for decay depends upon the type of atom. When the nucleus of a radionuclide spontaneously gives up its extra energy, that energy is called ionizing radiation. Ionizing radiation may take the form of alpha particles, beta particles, or gamma rays.
\end{abstract}

Keywords: Radioactive Materials, Uranium, Plutonium, Iodine, Polonium, Sodium, Fluorine Carbon, Cobalt, Lead, Radon

\section{Introduction}

All materials are made of atoms. Radioactive atoms are unstable; that is, they have too much energy. When radioactive atoms release their extra energy, they are said to decay. All radioactive atoms decay. After releasing all their excess energy, the atoms become stable and are no longer radioactive. The time required for decay depends upon the type of atom. It is not possible to predict exactly when a single radioactive atom will decay. However, the time required for half of a large number of identical radioactive atoms to decay has been determined. This time is called the half-life. When the nucleus of a radioactive isotope decays, emitting ionizing radiation, the nucleus is altered. It is transformed into another isotope which in many cases is a different element. This new isotope may be stable or unstable. If it is stable, the new isotope is not radioactive. If it is unstable, it also will decay, transforming its nucleus and emitting more ionizing radiation. Several decays may be required before a stable isotope is produced. In this paper we intend to introduce a number of radioactive elements found in nature and focus to explore their applications in various sciences.

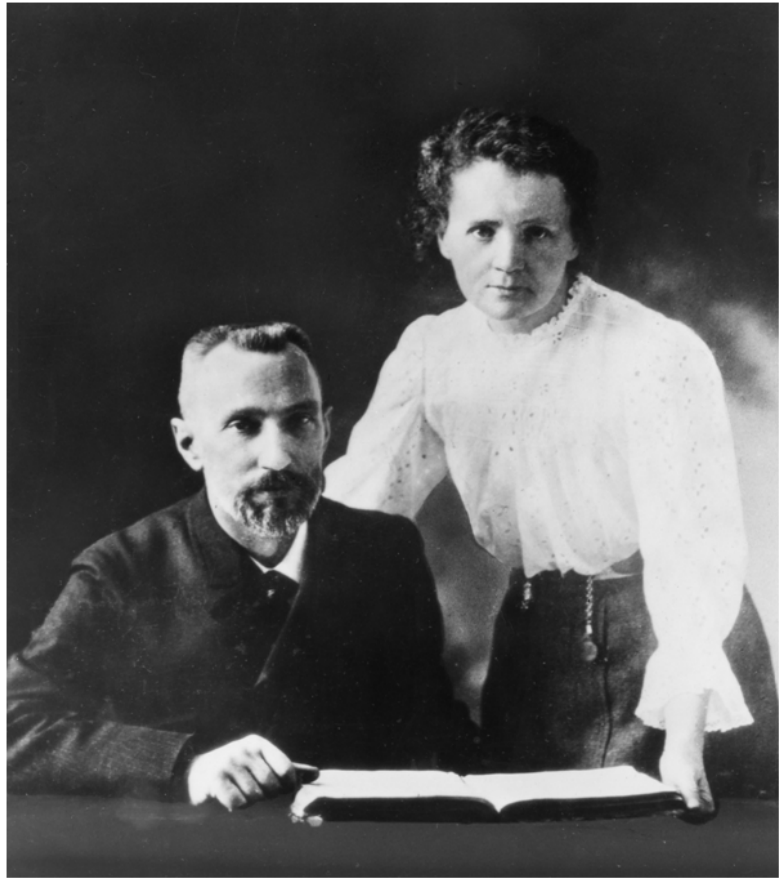

Figure 1. (pierre and marie) (www.espci.fr)

\section{2. $\mathrm{U}_{238}$ (Uranium-238)}

$\mathrm{U}_{238}$ is the most common isotope of uranium found in 
nature. It is not fissile, but is a fertile material: it can capture a slow neutron and after two beta decays become fissile plutonium-239. $U_{238}$ is fissionable by fast neutrons, but cannot support a chain reaction because inelastic scattering reduces neutron energy below the range where fast fission of one or more next-generation nuclei is probable. Doppler broadening of U-238's neutron absorption resonances, increasing absorption as fuel temperature increases, is also an essential negative feedback mechanism for reactor control.

Around $99.284 \%$ of natural uranium is uranium-238, which has a half-life of $1.41 \times 1017$ seconds $(4.468 \times 109$ years, or 4.468 billion years).[1] Depleted uranium has an even higher concentration of the $238 \mathrm{U}$ isotope, and even low-enriched uranium (LEU), while having a higher proportion of the uranium-235 isotope (in comparison to depleted uranium), is still mostly 238U. Reprocessed uranium is also mainly $238 \mathrm{U}$, with about as much uranium235 as natural uranium, a comparable proportion of uranium-236, and much smaller amounts of other isotopes of uranium such as uranium-234, uranium-233, and uranium-232. [2]. U-238 occur naturally in nearly all rock, soil, and water. U-238 is the most abundant form in the environment. U-235 can be concentrated in a process called "enrichment," making it suitable for use in nuclear reactors or weapons. Uranium 238, which is a chemical compound, can be used to breed $\mathrm{Pu}-239$ that can be used as fuel supply for a nuclear reactor. It can also be used for armor plating as well as in kinetic energy penetrators.

Because uranium decays by alpha particles, external exposure to uranium is not as dangerous as exposure to other radioactive elements because the skin will block the alpha particles. Ingestion of high concentrations of uranium, however, can cause severe health effects, such as cancer of the bone or liver. Inhaling large concentrations of uranium can cause lung cancer from the exposure to alpha particles. Uranium is also a toxic chemical, meaning that ingestion of uranium can cause kidney damage from its chemical properties much sooner than its radioactive properties would cause cancers of the bone or liver.

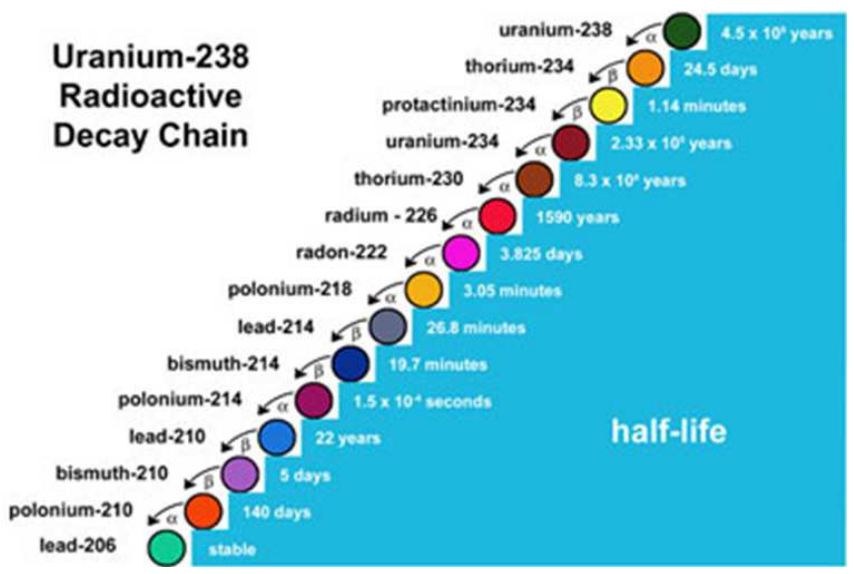

Figure 2. (geoinfo.nmt.edu)

\section{3. $\mathbf{U}_{235}$ (Uranium-235)}

Uranium metal highly enriched in uranium-235

Characterize:

Name, symbol: Uranium-235,235U

Neutrons: 143

Protons: 92

Nuclide data

Natural abundance: $0.72 \%$

Half-life:703,800,000 years

Parent isotopes: $235 \mathrm{~Pa}$

$235 \mathrm{~Np}$

$239 \mathrm{Pu}$

Decay products: $231 \mathrm{Th}$

Isotope mass: $235.0439299 \mathrm{u}$

Spin: 7/2-

Excess energy: $40914.062 \pm 1.970 \mathrm{keV}$

Binding energy: $1783870.285 \pm 1.996 \mathrm{keV}$

Decay mode Decay energy

Alpha: $4.679 \mathrm{MeV}$

Uranium-235 is an isotope of uranium making up about $0.72 \%$ of natural uranium. Unlike the predominant isotope uranium-238, it is fissile, i.e., it can sustain a fission chain reaction. It is the only fissile isotope that is a primordial nuclide or found in significant quantity in nature.Uranium235 has a half-life of 703.8 million years. It was discovered in 1935 by Arthur Jeffrey Dempster. Its (fission) nuclear cross section for slow thermal neutrons is about 504.81 barns. For fast neutrons it is on the order of 1 barn.[3] Most but not all neutron absorptions result in fission; a minority result in neutron capture forming uranium-236.

Heavy water reactors, and some graphite moderated reactors can use unenriched uranium, but light water reactors must use low enriched uranium because of light water's neutron absorption. Uranium enrichment removes some of the uranium-238 and increases the proportion of uranium-235. Highly enriched uranium, which contains an even greater proportion of U-235, is sometimes used in nuclear weapon design.

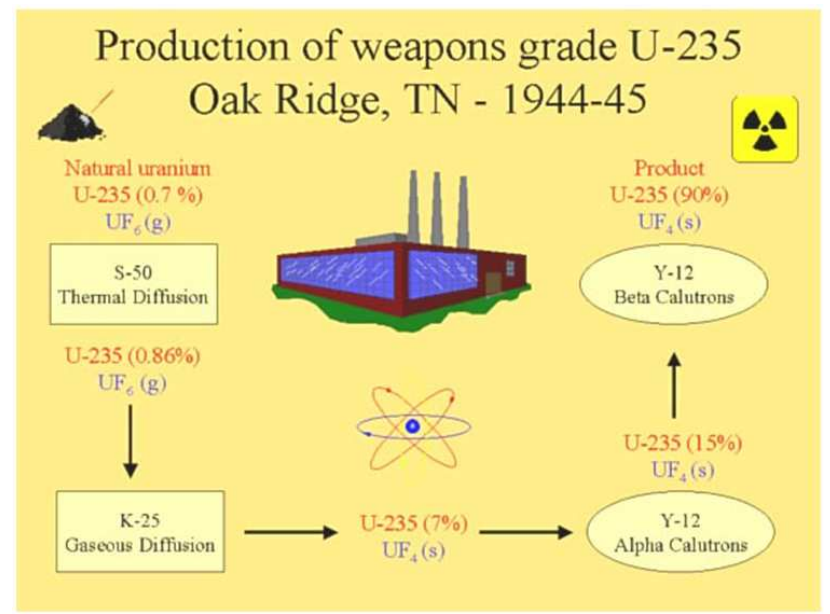

Figure 3. (chemcases.com) 
If at least one neutron from U-235 fission strikes another nucleus and causes it to fission, then the chain reaction will continue. If the reaction will sustain itself, it is said to be critical, and the mass of U-235 required to produce the critical condition is said to be a critical mass. A critical chain reaction can be achieved at low concentrations of $U$ 235 if the neutrons from fission are moderated to lower their speed, since the probability for fission with slow neutrons is greater. A fission chain reaction produces intermediate mass fragments which are highly radioactive and produce further energy by their radioactive decay. Some of them produce neutrons, called delayed neutrons, which contribute to the fission chain reaction. In nuclear reactors, the reaction is slowed down by the addition of control rods which are made of elements such as boron, cadmium, and hafnium which can absorb a large number of neutrons. In nuclear bombs, the reaction is uncontrolled and the large amount of energy released creates a nuclear explosion.

Given enough fuel, an atomic explosion can result. On the other hand, if the amount of available fuel is carefully controlled, along with other factors, the output of energy may serve useful purposes. Although the radioactive fuel used may vary, the production of energy by neutronemitting processes is used in nuclear power facilities that can heat homes and factories. Although the fission of atoms of Uranium 235 produces nuclear wastes that present challenges not to be minimized, unlike fuels such as coal, oil or wood, nuclear energy does not unavoidably bring along with it the age-old problems of polluted air or polluted water.

\section{Pu-239 (Plutonium-239)}

Plutonium-239 is an isotope of plutonium. Plutonium239 is the primary fissile isotope used for the production of nuclear weapons, although uranium-235 has also been used. Plutonium-239 is also one of the three main isotopes demonstrated usable as fuel in nuclear reactors, along with uranium-235 and uranium-233. Plutonium-239 has a halflife of 24,100 years. The nuclear properties of plutonium239 , as well as the ability to produce large amounts of nearly pure $\mathrm{Pu}-239$ more cheaply than highly enriched weapons-grade uranium-235, led to its use in nuclear weapons and nuclear power stations. The fissioning of an atom of uranium-235 in the reactor of a nuclear power plant produces two to three neutrons, and these neutrons can be absorbed by uranium-238 to produce plutonium-239 and other isotopes. Plutonium-239 can also absorb neutrons and fission along with the uranium-235 in a reactor. Of all the common nuclear fuels, $\mathrm{Pu}-239$ has the smallest critical mass. A spherical untampered critical mass is about $11 \mathrm{~kg}$ (24.2 lbs),[4] $10.2 \mathrm{~cm}$ (4") in diameter. Using appropriate triggers, neutron reflectors, implosion geometry and tampers, this critical mass can be reduced by more than twofold.

\section{Common uranium isotope 238 absorbs a neutron to} become uranium 239

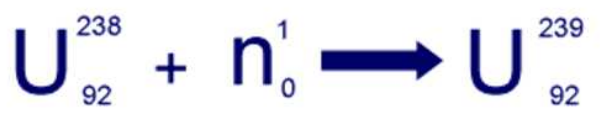

This is a beta emitter - it decays to produce neptunium 239

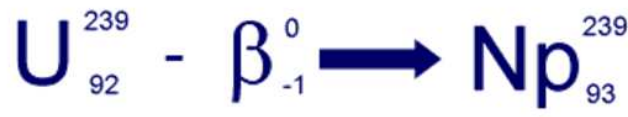

which in turn decays to produce plutonium 230

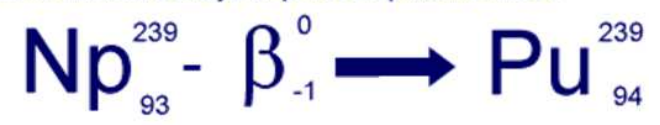

Figure 4. (www.cyberphysics.co.uk)

\section{Iodine 131}

Iodine-131 (131I), also called radioiodine, is an important radioisotope of iodine discovered by Glenn Seaborg and John Livingood in 1938 at the University of California, Berkeley.[5] It has a radioactive decay half-life of about eight days. It is associated with nuclear energy, medical diagnostic and treatment procedures, and natural gas production. It also plays a major role as a radioactive isotope present in nuclear fission products, and was a significant contributor to the health hazards from open-air atomic bomb testing in the $1950 \mathrm{~s}$, and from the Chernobyl disaster, as well as being a large fraction of the contamination hazard in the first weeks in the Fukushima nuclear crisis. This is because I-131 is major uranium, plutonium fission product, comprising nearly $3 \%$ of the total products of fission (by weight). See fission product yield for a comparison with other radioactive fission products. I-131 is also a major fission product of uranium233, produced from thorium.Iodines is among the most widely used radionuclide's, mostly in the medical field. Because of its short half-life and useful beta emission, iodine-131 is used extensively in nuclear medicine. Its tendency to collect in the thyroid gland makes iodine especially useful for diagnosing and treating thyroid problems. Iodine-123 is widely used in medical imaging, and I-124 is useful in immunotherapy. Iodine's chemical properties make it easy to attach to molecules for imaging studies. It is useful in tracking the metabolism of drugs or compounds, or for viewing structural defects in various organs, such as the heart. A less common isotope, iodine125 , is sometimes used to treat cancerous tissue.Iodine-129 has little practical use, but may be used to check some radioactivity counters in diagnostic testing laboratories. 


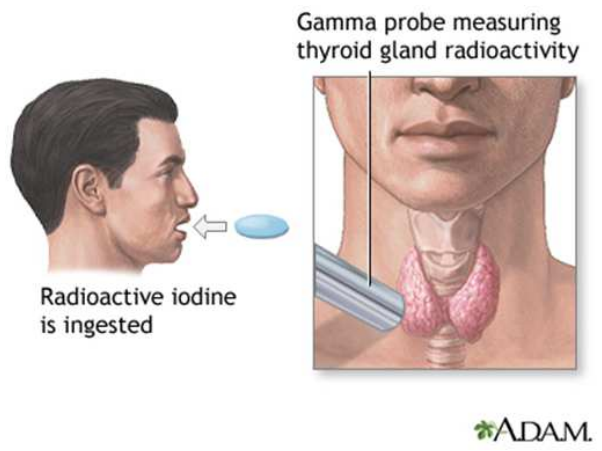

Figure 5. (www.scripps.org)

\section{Polonium 210}

Polonium was discovered by Marie Sklodowska-Curie and Pierre Curie in 1898 and was named after Marie's native land of Poland (Latin: Polonia). This element was the first one discovered by them while they were investigating the cause of pitchblende radioactivity. Polonium ( $\mathrm{Po}$ ) has 33 isotopes, all of which are radioactive, with between 186 and 227 nucleons. 210Po with a half-life of 138.376 days has the longest half-life of naturally occurring polonium. 209Po with a half-life of 103 years has the longest half-life of all isotopes of polonium. 209Po and 208Po (half-life 2.9 years) can be made through the alpha, proton, or deuteron bombardment of lead or bismuth in a cyclotron. Being produced during the decay of naturally occurring uranium-238, polonium-210 is widely distributed in small amounts in the earth's crust. Although it can be produced by the chemical processing of uranium ores or minerals, uranium ores contain less than $0.1 \mathrm{mg}$ Po-210 per ton. Because Po-210 is produced from the decay of radon222 gas, it can be found in the atmosphere from which it is deposited on the earth's surface. Although direct root uptake by plants is generally small, Po-210 can be deposited on broad-leaved vegetables. Deposition from the atmosphere on tobacco leaves results in elevated concentrations of Po-210 in tobacco smoke. There are tiny amounts of Po-210 in our bodies. Po-210 can be manufactured artificially by irradiating stable bismuth-209 with thermal neutrons resulting in the formation of radioactive $\mathrm{Bi}-210$, which decays (half-life 5 days) into Po210. Polonium may now be made in milligram amounts in this procedure which uses high neutron fluxes found in nuclear reactors. Only about 100 grams are produced each year, making polonium exceedingly rare. Po-210 is used in neutron sources (where it is mixed or alloyed with beryllium). It is also used in devices that eliminate static electricity in machinery where it can be caused by processes such as paper rolling, manufacturing sheet plastics, and spinning synthetic fibres. Brushes containing Po-210 are used to remove accumulated dust from photographic films and camera lenses. Static eliminators typically contain from one to tens of $\mathrm{GBq}$ of radioactivity.Po-210 emits so many alpha particles each second that the energy released from one gram is 140 watts, and a capsule containing about half a gram will spontaneously reach a temperature of $500^{\circ} \mathrm{C}$. As a result it has been used as a lightweight heat source to power thermoelectric cells in satellites. A Po-210 heat source was also used in each of the Lunokhod rovers deployed on the surface of the Moon, to keep their internal components warm during the lunar nights. However, because of its short half-life Po-210 cannot provide power for long-term space missions and has been phased out of use in this application. Polonium is not subject to IAEA safeguards.

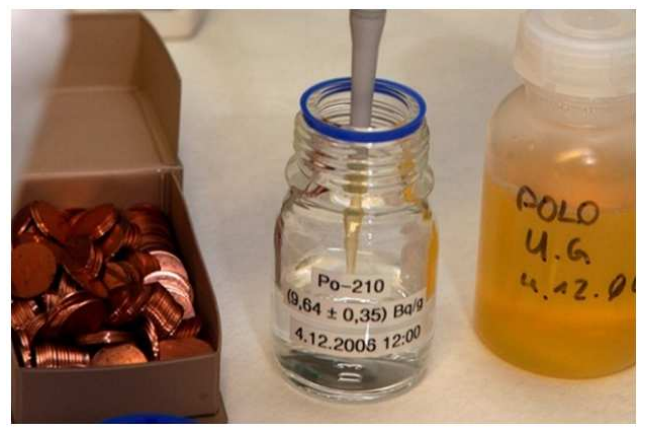

Figure 6. (www.aljazeera.com)

\section{Sodium 24 (Na 24)}

There are twenty recognized isotopes of sodium, ranging from $18 \mathrm{Na}$ to $37 \mathrm{Na}$ and two isomers $(22 \mathrm{~m} \mathrm{Na}$ and $24 \mathrm{~m}$ $\mathrm{Na}$ ). $23 \mathrm{Na}$ is the only stable (and the only primordial) isotope. As such, it is considered a monoisotopic element and it has a standard atomic mass: 22.98976928(2) u. Sodium has two radioactive cosmogony isotopes (22 Na, half-life $=2.605$ years; and $24 \mathrm{Na}$, half-life $\approx 15$ hours). With the exception of those two, all other isotopes have half-lives under a minute, most under a second. The least stable is $18 \mathrm{Na}$, with a half-life of $1.3(4) \times 10-21$ seconds. Acute neutron radiation exposure (e.g., from a nuclear criticality accident) converts some of the stable $23 \mathrm{Na}$ in human blood plasma to $24 \mathrm{Na}$. By measuring the concentration of this isotope, the neutron radiation dosage to the victim can be computed.Sodium-22 is a positronemitting isotope with a remarkably long half-life. It is used to create test-objects and point-sources for positron emission tomography.

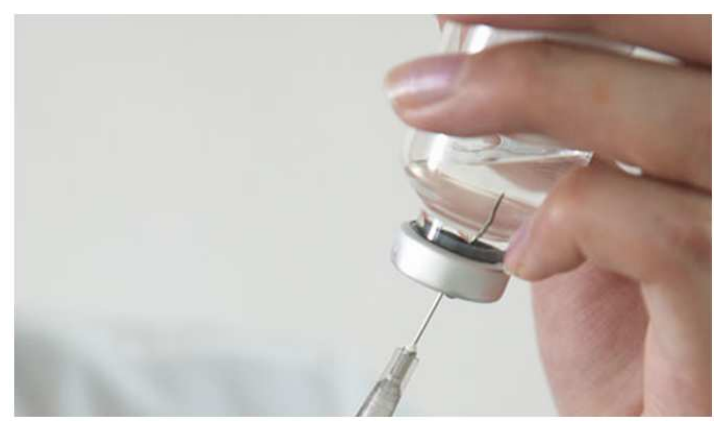

Figure 7. (www.theguardian.com) 


\subsection{Advantages of Using Sodium-24}

Sodium-24 is a very effective means of detecting leakages in the industry, although suffering from some minor disadvantages, it does not detract from its ability to efficiently provide assistance to detection of leakages in the industry and is certainly valuable assistance in the industry.

- Sodium-24 is non-toxic to humans or animals and although it may form organic compounds absorbed into the blood stream, this will not unduly affect the health of humans or animals absorbing Sodium-24 in general due to a variety of other factors such as its life span and low intensity emission of gamma radiation.

- Sodium-24 is a beta and low intensity gamma emitter, no radiation will be detected if the pipe has not sprung a leak as it will not emit radiation penetrating the pipe's thick walls, (beta radiation may be blocked by something as thin as paper), in most instances beta radiation cannot penetrate the skin.

- Sodium-24 being a beta radiator may potentially be harmful to animal and humans if ingested or directly injected into the bloodstream. However having a short life span of roughly 15 hours, Sodium-24 does not pose a direct threat to any humans or animals.

\subsection{Disadvantages of Using Sodium-24}

- $\quad$ Sodium-24 is non-naturally occurring radioisotope, it relies on commercial production in nuclear reactors and poses the same threats that nuclear reactors have. I.e. nuclear fallout and disasters.

- Having a short lifespan also prevents the area of testing from being very far from the source.

- Radioisotopes have long term effects and implications to workers handling the substance, without proper care in transportation and handling Sodium 24 can cause biological damage, which may result in tissue damage or cancer.

\section{Gold 128}

Gold (Au) has one stable isotope, ${ }^{197} \mathrm{Au}$, and 36 radioisotopes, with ${ }^{195} \mathrm{Au}$ being the most stable with a halflife of 186 days. Gold is currently considered the heaviest monoisotopic element (bismuth formerly held that distinction, but bismuth-209 has been found to be slightly radioactive).Standard atomic mass: 196.966569(4) u

Inside coker units at oil refineries, Gold-198 is used to study the hydrodynamic behavior of solids in fluidized beds and can also be used to quantify the degree of fouling of bed internals. [6] Gold-198 is a beta emitter with range in tissue of about $11 \mathrm{~mm}$ and half life 2.7 days. It is used in some cancer treatments and for treating other diseases.[7][8] Gold-198 nanoparticles are being investigated as an injectable treatment for prostate cancer.[9]

\section{Fluorine}

Although fluorine $(\mathrm{F})$ has 18 known isotopes from $14 \mathrm{~F}$ to $31 \mathrm{~F}$ and one isomer $(18 \mathrm{mF})$, only one of these isotopes is stable, that is, fluorine-19; as such, it is a monoisotopic element. The longest-lived radioisotope is $18 \mathrm{~F}$ with a halflife of 109.771 minutes. All other isotopes have half-lives under a minute, the majority under a second, making fluorine a mononuclidic element as well. The least stable isotope is $15 \mathrm{~F}$, whose half-life is $4.1 \times 10-22$ seconds, corresponding to a spectral linewidth of about $1 \mathrm{MeV}$. Only 14F has an unknown half-life.

The nuclide ${ }^{18} \mathrm{~F}$ is the radionuclide of fluorine with the longest half-life, 109.771 minutes, allowing it to serve commercially as an important source of positrons. Its major use is for the production of the radiopharmaceutical fludeoxyglucose for positron emission tomography scanning in medicine.

Fluorine-19, the only stable isotope of fluorine. Its abundance is $100 \%$; no other isotopes of fluorine exist in significant quantities. Its binding energy is $147801 \mathrm{keV}$. Fluorine-19 is NMR-active, so it is used in fluorine-19 NMR spectroscopy.

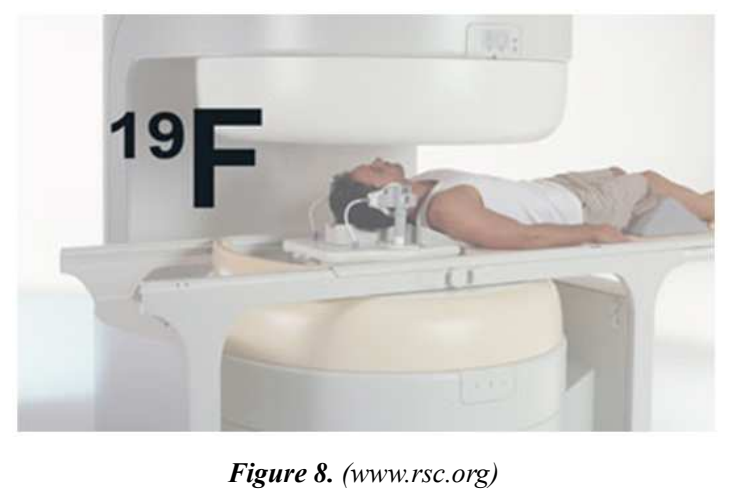

Fluorine-20, is one of the more unstable isotopes of fluorine. It has a half-life of 11.07 seconds and undergoes beta decay, transforming into its daughter nuclide $20 \mathrm{Ne}$. Its specific radioactivity is $1.885 \times 109 \mathrm{TBq} / \mathrm{g}$ and has a lifetime of 15.87 seconds.

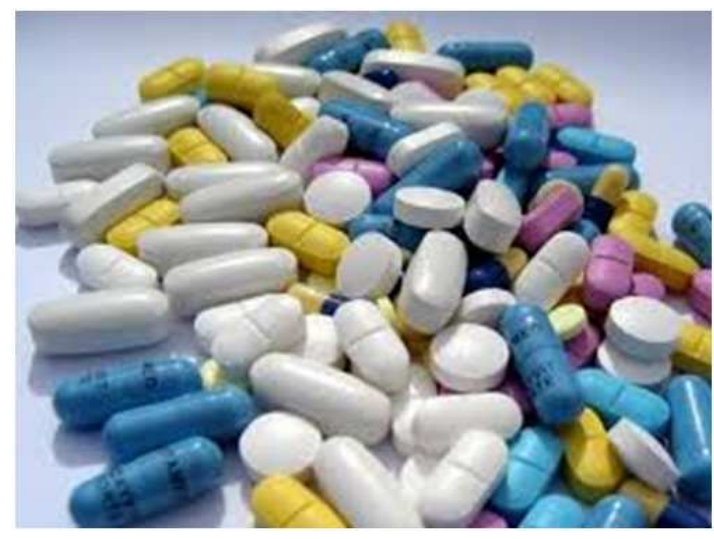

Figure 9. (soumyajitblog.wordpress.com) 
Fluorine-21, as with fluorine-20, is also one of the more unstable isotopes of this element. It has a half-life of 4.158 seconds. It undergoes beta decay as well which leaves behind a daughter nuclei of ${ }^{21} \mathrm{Ne}$. Its specific radioactivity is $4.78 \times 10^{9} \mathrm{TBq} / \mathrm{g}$.

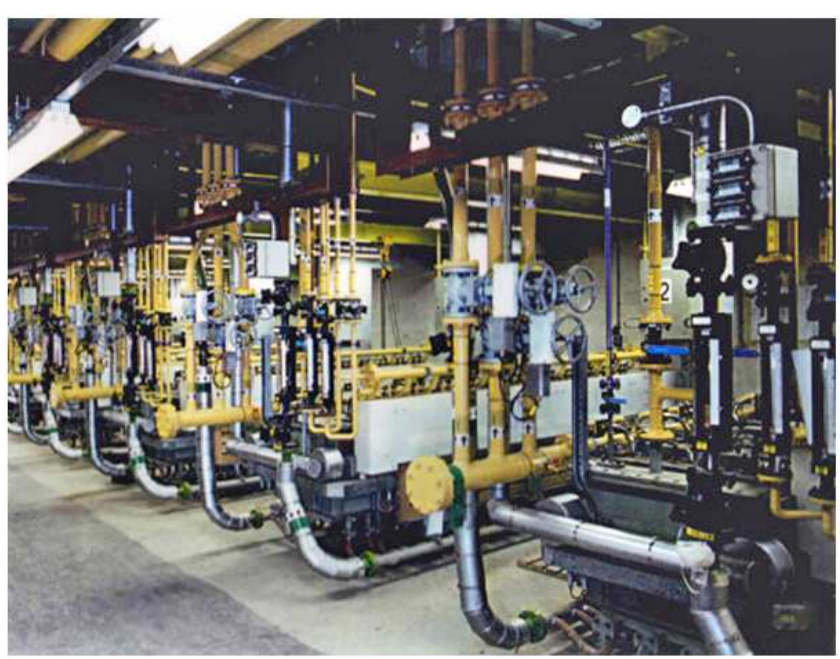

Figure 10. (en.wikipedia.org)

\section{C14 (Carbon 14)}

Carbon- $14,{ }^{14} \mathrm{C}$, or radiocarbon, is a radioactive isotope of carbon with a nucleus containing 6 protons and 8 neutrons. Its presence in organic materials is the basis of the radiocarbon dating method pioneered by Willard Libby and colleagues (1949) to date archaeological, geological and hydro geological samples[10]. Carbon-14 was discovered on 27 February 1940, by Martin Kamen and Sam Ruben at the University of California Radiation Laboratory in Berkeley. Its existence had been suggested by Franz Kurie in 1934[11].There are three naturally occurring isotopes of carbon on Earth: $99 \%$ of the carbon is carbon$12,1 \%$ is carbon-13, and carbon-14 occurs in trace amounts, i.e., making up about 1 part per trillion $(0.0000000001 \%)$ of the carbon in the atmosphere. The half-life of carbon-14 is $5,730 \pm 40$ years. [12]Carbon-14 decays into nitrogen-14 through beta decay. [13]The primary natural source of carbon-14 on Earth is cosmic ray action upon nitrogen in the atmosphere, and it is therefore a cosmogony nuclide. However, open-air nuclear testing between 1955-1980 contributed to this pool. The different isotopes of carbon do not differ appreciably in their chemical properties. This is used in chemical and biological research, in a technique called carbon labeling: carbon-14 atoms can be used to replace nonradioactive carbon, in order to trace chemical and biochemical reactions involving carbon atoms from any given organic compound. Another isotope, carbon-14, is useful in studying abnormalities of metabolism that underlie diabetes, gout, anemia, and acromegaly. in 1948, when Willard F. Libby, at the University of Chicago, developed the process of radioactive carbon dating. In this method, the activity of radioactive carbon (carbon-14) present in bones, wood, or ash found in archaeological sites is measured. A notable example is the use of carbon-14 (14C). Today, a number of oceanographic laboratories make carbon-14 measurements of oceanic dissolved carbon for the study of mixing and transport processes in the deep ocean. In 1961 the isotope carbon-12 was selected to replace oxygen as the standard relative to which the atomic weights of all the other elements are measured; carbon-14, which is radioactive, is the isotope used in radiocarbon dating and radio labeling. Another medically important radioactive isotope is carbon-14, which is useful in studying abnormalities of metabolism that underlie diabetes, gout, anemia, and acromegaly

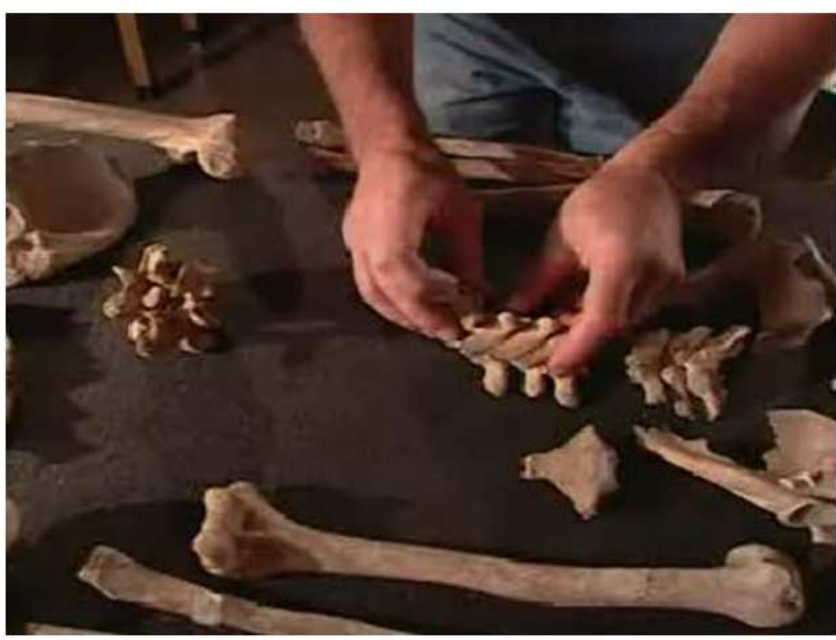

Figure 11. (science.howstuffworks.com.)

\section{Cobalt 60}

In 1735, a Swedish scientist, George Brandt, demonstrated that a blue color common in colored glass was caused by a new element, cobalt. Previously, people thought that bismuth, which occurs in nature with cobalt, was the cause. Radioactive cobalt- 60 was discovered by Glenn T. Seaborg and John Livingood at the University of California - Berkeley in the late 1930's. Non radioactive cobalt occurs naturally in various minerals, and has been used for thousands of years to impart blue color to ceramic and glass. The radionuclide, cobalt-60, is produced for commercial use in linear accelerators. It is also produced as a by-product of nuclear reactor operations, when structural materials, such as steel, are exposed to neutron radiation. Cobalt-60 is used in many common industrial applications, such as in leveling devices and thickness gauges, and in radiotherapy in hospitals. Large sources of cobalt-60 are increasingly used for sterilization of spices and certain foods. The powerful gamma rays kill bacteria and other pathogens, without damaging the product. After the radiation ceases, the product is not left radioactive. This process is sometimes called "cold pasteurization. Cobalt-60 is also used for industrial radiography, a process similar to an x-ray, to detect structural flaws in metal parts. One of its uses is in a medical device for the precise treatment of 
otherwise inoperable deformities of blood vessels and brain tumors. Radionuclides, such as cobalt-60, that are used in industry or medical treatment are encased in shielded metal containers or housings, and are referred to as radiation 'sources.' The shielding keeps operators from being exposed to the strong radiation. Cobalt- 60 can also be released to the environment through leaks or spills at nuclear power plants, and in solid waste originating from nuclear power plants. Nuclear Regulatory Commission regulations allow small amounts of cobalt- 60 to be released into the air, or poured down drains as part of a liquid.

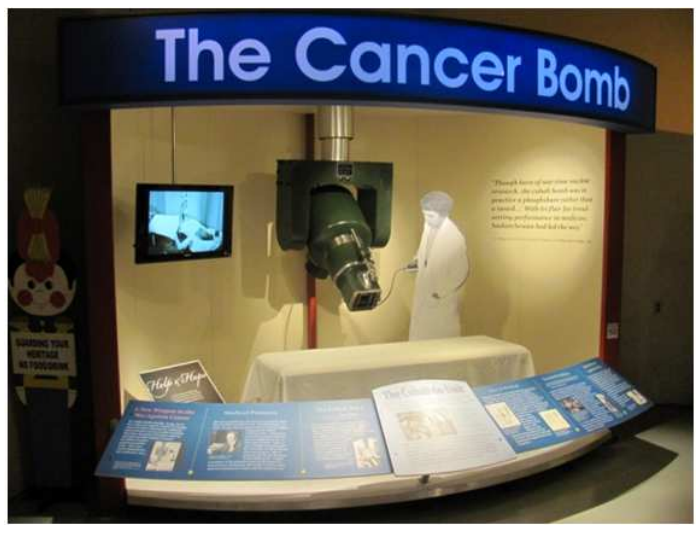

Figure 12. (www.ckom.com)

\section{2. $\mathrm{Pb}$ (Lead)}

Lead occurs naturally on Earth exclusively in the form of four isotopes: lead-204, -206, -207, and -208. [14]All four can be radioactive as the hypothetical alpha decay of any would be exothermic, but the lower half-life limit has been put only for lead-204: over $1.4 \times 10^{17}$ years. [15]This effect is, however, so weak that natural lead poses no radiation hazard. Three isotopes are also found in three of the four major decay chains: lead-206, -207 and -208 are final decay products of uranium-238, uranium-235, and thorium-232, respectively. Since the amounts of them in nature depend also on other elements' presence, the isotopic composition of natural lead varies by sample: in particular, the relative amount of lead-206 varies between $20.84 \%$ and $27.78 \%$.[14]Aside from the stable ones, thirty-four radioisotopes have been synthesized: they have mass numbers of 178-215. [15] Lead-205 is the most stable radioisotope of lead, with a half-life of over $10^{7}$ years. 47 nuclear isomers (long-lived excited nuclear states), corresponding to 24 lead .isotopes, have been characterized. The most long-lived isomer is lead-204m2 (half-life of about 1.1 hours). Lead is used in building construction, lead-acid batteries, bullets and shot, weights, as part of solders, pewters, fusible alloys, and as a radiation shield. Lead has the highest atomic number of all of the stable elements, although the next higher element, bismuth, has a half-life that is so long (over one billion times the estimated age of the universe) that it can be considered stable. Its four stable isotopes have 82 protons, a magic number in the nuclear shell model of atomic nuclei. The isotope $208 \mathrm{~Pb}$ is magic. If ingested; lead is poisonous to animals, including humans. It damages the nervous system and causes brain disorders. Excessive lead also causes blood disorders in mammals. Like the element mercury, another heavy metal, lead is a neurotoxin that accumulates both in soft tissues and the bones. Lead poisoning has been documented from ancient Rome, ancient Greece, and ancient China.

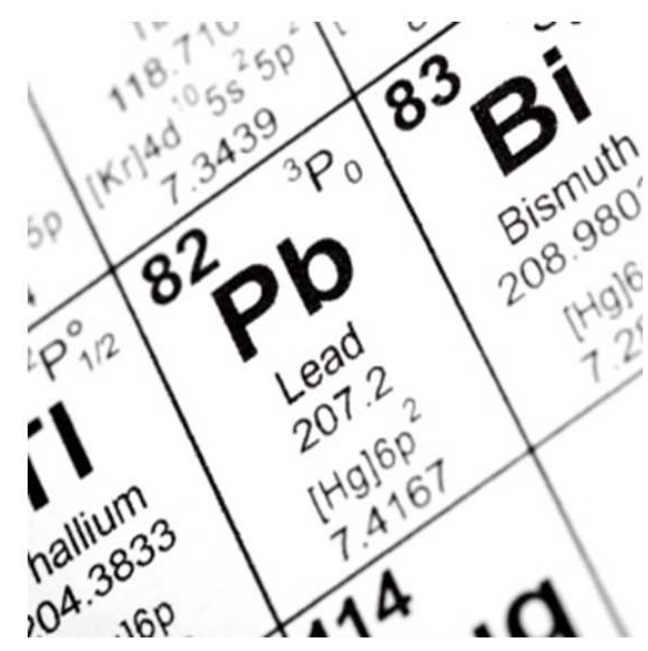

Figure 13. (science.howstuffworks.com)

\section{Radon (Rn)}

Radon is a chemical element with symbol Rn and atomic number 86. It is a radioactive, colorless, odorless, tasteless[16] noble gas, occurring naturally as an indirect decay product of uranium or thorium. Its most stable isotope, ${ }^{222} \mathrm{Rn}$, has a half-life of 3.8 days. Radon is one of the densest substances that remains a gas under normal conditions. It is also the only gas under normal conditions that only has radioactive isotopes, and is considered a health hazard due to its radioactivity. Intense radioactivity has also hindered chemical studies of radon and only a few compounds are known.Radon is formed as one intermediate step in the normal radioactive decay chains, through which thorium and uranium slowly decay into lead. Thorium and uranium are the two most common radioactive elements on earth; they have been around since the earth was formed. Their naturally occurring isotopes have very long half-lives, on the order of billions of years. Thorium and uranium, their decay product radium, and its decay product radon, will therefore continue to occur for tens of millions of years at almost the same concentrations as they do now. [17] As radon itself decays, it produces new radioactive elements called radon daughters or decay products. Unlike the gaseous radon itself, radon daughters are solids and stick to surfaces, such as dust particles in the air. If such contaminated dust is inhaled, these particles can stick to the airways of the lung and increase the risk of developing lung cancer. [18]Unlike all the other intermediate elements in the aforementioned decay chains, radon is gaseous, and is 
thus easily inhaled. Thus, even in this age of nuclear reactors, naturally-occurring radon is responsible for the majority of the public exposure to ionizing radiation. It is often the single largest contributor to an individual's background radiation dose, and is the most variable from location to location. Despite its short lifetime, some radon gas from natural sources can accumulate to far higher than normal concentrations in buildings, especially in low areas such as basements and crawl spaces due to its heavy nature. It can also be found in some spring waters and hot springs [19].

Radon was sometimes used in hospitals to treat cancer and was produced as needed and delivered in sealed gold needles. Radon is used in hydrologic research, because of it's rapid loss to air. It is also used in geologic research and to track air masses. Background levels of radon in outside air are generally quite low, but in indoor locations radon levels in air may be higher. In homes, schools and buildings radon levels are increased because radon enters the buildings through cracks in the foundations and basements. Exposure to high levels of radon through breathing air is known to cause lung diseases. When long-term exposure occurs radon increases the chances of developing lung cancer. Radon can only cause cancer after several years of exposure.

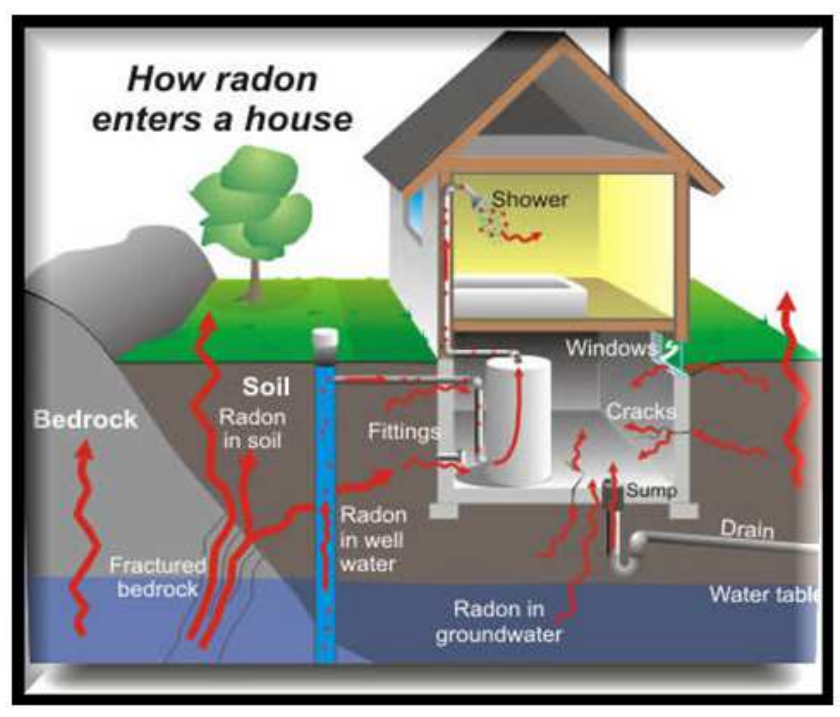

Figure 14. (imgarcade.com)

\section{Discussion}

Long-lived radioactive elements such as uranium, thorium and potassium and any of their decay products, such as radium and radon are examples of NORM. These elements have always been present in the Earth's crust and atmosphere. The term NORM exists also to distinguish 'natural radioactive material' from anthropogenic sources of radioactive material, such as those produced by nuclear power and used in nuclear medicine, where incidentally the radioactive properties of a material maybe what make it useful. However from the perspective of radiation doses to people, such a distinction is completely arbitrary. Exposure to naturally occurring radiation is responsible for the majority of an average person's yearly radiation dose (see also Nuclear Radiation and Health Effects paper) and is therefore not usually considered of any special health or safety significance. However certain industries handle significant quantities of NORM, which usually ends up in their waste streams. Over time, as potential NORM hazards have been identified, these industries have increasingly become subject to monitoring and regulation. However, there is as yet little consistency in NORM regulations among industries and countries. This means that material which is considered radioactive waste in one context may not be considered so in another. Also, that which may constitute low-level waste in the nuclear industry might go entirely unregulated in another industry.

\section{References}

[1] Mcclain, D.E.; A.C. Miller, J.F. Kalinich (December 20, 2007). "Status of Health Concerns about Military Use of Depleted Uranium and Surrogate Metals in ArmorPenetrating Munitions" (pdf). NATO. Retrieved November 14, 2010.

[2] Nuclear France: Materials and sites. "Uranium from reprocessing"

[3] Some Physics of Uranium

[4] FAS Nuclear Wepons Design FAQ, Accessed 2010-9-2

[5] "UW-L Brachy Course". wikifoundry. April 2008. Retrieved 2014-04-11.

[6] Rhodes, Richard (1995). Dark sun: The making of the hydrogen bomb. New York: Simon \& Schuster. ISBN 0-68480400-X.

[7] "Nanoscience and Nanotechnology in Nanomedicine: Hybrid Nanoparticles In Imaging and Therapy of Prostate Cancer". Radiopharmaceutical Sciences Institute, University of Missouri-Columbia.

[8] Hainfeld, James F.; Dilmanian, F. Avraham; Slatkin, Daniel N.; Smilowitz, Henry M. (2008). "Radiotherapy enhancement with gold nanoparticles". Journal of Pharmacy $\begin{array}{llll}\text { and Pharmacology } 60 & \text { (8): 977-85. Doi: }\end{array}$ 10.1211/jpp.60.8.0005. PMID 18644191.

[9] "Green Tea and Gold Nanoparticles Destroy Prostate Tumors". 2012.

[10] Waptstra, A.H.; Audi, G. and Thibault, C. "AME atomic mass evaluation 2003". Retrieved 2007-06-03.

[11] Kamen, Martin D. (1963). "Early History of Carbon-14: Discovery of this supremely important tracer was expected in the physical sense but not in the chemical sense". Science 140 (3567): 584-590. Bibcode:1963Sci.140.584K. Doi:10.1126/science.140.3567.584. PMID 17737092.

[12] Godwin, H (1962). "Half-life of radiocarbon". Nature 195 (4845): $\quad 984 . \quad$ Bibcode:1962Natur.195..984G. Doi:10.1038/195984a0 
[13] "What is carbon dating?". National Ocean Sciences Accelerator Mass Spectrometry Facility. Retrieved 2007-0611

[14] Polyanskiy 1986, p. 16.

[15] G. Audi, A. H. Wapstra, C. Thibault, J. Blachot and O. Bersillon (2003). "The NUBASE evaluation of nuclear and decay properties". Nuclear Physics a 729 (1): 3-128. Bibcode:2003NuPhA.729...3A. Doi:10.1016/j.nuclphysa.2003.11.001..

[16] "A Citizen's Guide to Radon | Radon | US EPA". Epa.gov. 2010-08-05. Retrieved 2012-04-28.
[17] Toxicological profile for radon, Agency for Toxic Substances and Disease Registry, U.S. Public Health Service, In collaboration with U.S. Environmental Protection Agency, December 1990.

[18] "Public Health Fact Sheet on Radon - Health and Human Services". Mass.Gov. Archived from the original on 201111-21. Retrieved 2011-12-04.

[19] "Facts about Radon". Facts about. Retrieved 2008-09-07. 